\section{Computing dynamic organizational proximity: The PROXTIME computer program}

\author{
KENNETH K. KIRSTE \\ System Development Corporation \\ Santa Monica, California 90406 \\ and \\ PETER R. MONGE \\ Michigan State University, East Lansing, Michigan 48824
}

Psychologists have long been interested in the role that proximity plays in determining various aspects of human behavior, such as communication and interaction (Festinger, Schacter, \& Back, 1950; Newcomb, 1961), attraction (Kahn \& McGaughey, 1977), and impression formation (Tesch, 1979). During the past decade, organizational psychologists have begun to explore the relationship of proximity to a variety of variables important to the work place, such as performance (Conrath, 1973), job satisfaction (Quinn, 1977), and organizational commitment (Conrath, 1973; Monge \& Kirste, 1980).

Monge and Kirste (1980) developed a definition and operationalization of proximity that is more appropriate for research in organizational contexts than are earlier methods developed from interpersonal studies. The definition contains three elements that distinguish proximity in organizations from the same construct in interpersonal settings: (1) that a person's proximity is usefully conceptualized in terms of meaningful work spaces that facilitate or inhibit the opportunity for faceto-face communication, (2) that each person in an organization is proximate to multiple others, and (3) that a person's proximity to these multiple others is dynamic and changes over time. The index developed for this definition provides the conditional probability of an individual's spending time in the same physical locations with each other person in the organization. It varies from 0 , representing complete isolation from all other individuals, to 1 , representing continuously sharing the same work space with all other individuals. A second index, indicating the proximity of each person to all others in the organization, can also be computed.

Recently, Monge, White, Eisenberg, Miller, and Kirste (Note 1) reported research on the dynamics of "organizational proximity," an aggregate-level measure for an entire organization, which is defined as the degree to which people within an organization share the same physical locations during the same periods of time; there is assumed to be both the opportunity and psychological obligation to engage in face-to-face communication. Data were gathered in 15 -min intervals for 1 workweek on the amount of time that employees spent in the different "locations" that constituted the entire organization. A single measure of organizational proximity indicating the "density" of people across all locations was computed for each of the 205 time periods for the week and subjected to time-series analysis. The results indicated both a significant daily variation in the congregation and isolation of people and a systematic day-to-day pattern.

Since more than 50,000 data points are required to represent 250 people in different locations during a workweek, it is extremely helpful to have access to a computer program that computes the values of organizational proximity. Data collection typically requires that all individuals in the organization record the locations where they spend their time for predetermined intervals (e.g., each $15 \mathrm{~min}$ ) over some period of time (e.g., a day, week). PROXTIME, the computer program described in this report, can be used to compute organizational proximity values for each (user-defined) time interval, as well as individual proximity values for each person. These values may be utilized in other analyses to test relevant theories and hypotheses.

\section{PROXTIME}

The description of PROXTIME that follows is organized into seven sections: a general description, the structure of PROXTIME runs, error checking, program limitations, PROXTIME calculations, hardware and transportability, and program availability.

General Description. PROXTIME calculates a single value for each 15 -min period representing how proximate an average individual is to all others in the organization at that time. The mathematics for calculating the proximity value are straightforward. The use of PROXTIME is important because the number of calculations is extensive. As currently written, PROXTIME can handle up to 250 individuals at 15 -min intervals over a 5-day, 10-h/day (0800-1800 h) workweek.

Structure of PROXTIME Runs. PROXTIME requires input in card format. The data input must consist of a header card consisting of all zeros, data cards, and a trailer card consisting of all nines. The data cards contain: (1) a three-digit unique identifier for each person, (2) a single-digit identifier for the day of the week, (3) a three-digit code for each location, (4) a four-digit start time (military time) for each location, and (5) a four-digit end time (military time) for each location. Up to five locations and their accompanying start and end times can appear on a single data card. The person and day identifiers must be repeated for data cards containing additional locations and times.

Error Checking. PROXTIME performs error checking to insure the integrity of the values calculated. Whenever PROXTIME detects a problem, error messages are output identifying the nature of the problem and the action PROXTIME is taking. Most errors result in the output of a message, and processing continues. PROXTIME checks for and handles the following errors: (1) a valid header card (if not found, it aborts), (2) the day code on each data card to determine if it is within limits (if it is not, the card image is printed and the entire 
card is ignored; processing continues), and (3) the start times and end times for each location to verify that the reported start time precedes the reported end time. Should PROXTIME locate a start time that is greater than an end time, it outputs the identifier for the individual and a warning message. PROXTIME ignores all data for this individual with the start/end time discrepancy and continues processing. PROXTIME does not contain code to detect erroneously punched data cards. Users must check the detection capabilities of the operating system under which they are operating PROXTIME to determine what error-checking capabilities are in force.

Limitations. (1) PROXTIME is coded to process data for Monday-Friday (Codes 1.5, even though it will accept Codes 6 and 7 (which denote Saturday and Sunday). Minor changes to the program can be made to expand PROXTIME for 7-day calculations.

(2) PROXTIME is coded to process up to 250 individual identifiers. Changes to the sizes of the arrays and to several program loops would be required to expand the number of persons the program will process.

(3) The program is coded to calculate proximity between the hours of 0800 and 1800 . Minor changes to several program loops are required to expand or contract the span of hours considered for each day. However, extensive alteration is required for PROXTIME to be able to handle a 24-h day. Therefore, alterations to the limits of start/end time values must not allow a span to cross midnight without considerable alteration to the program.

(4) The program is coded to handle up to 10,000 location/time combinations. (This is an average of eight different locations per day for each of a maximum of 250 people and is greater when fewer than 250 people are tracked.) PROXTIME is capable of handling up to 1,000 different start/end times reported for 10 different locations or 100 different start/end times reported for 100 different locations, or any other combination that does not exceed 10,000 entries. However, the data card is set up to accept a three-digit location identifier and codes in the 700,800 , and 900 series are used to control PROXTIME operations, effectively limiting the number of locations to fewer than 699. Minor changes to array sizes and to loop controls are required to expand the number of locations/times the program will process.

(5) PROXTIME handles 15-min intervals. Minor changes to the code are required to expand or contract this interval.

(6) PROXTIME is coded to print out a proximity value indicating how proximate all the individuals are at each time period. During the calculation of this value, it also calculates how proximate each individual is to all others. Minor changes to the program will allow the output of individual proximity values per each time period.

(7) PROXTIME does not output an average value indicating how proximate an individual is to all others across an entire day or week. Additional program code and space are required to store the individual proximity values and average them at the conclusion of other calculations.

(8) The program is not coded to output an average value for the proximity of all individuals across each day and/or the entire week. Additional program code and space are required to add this capability.

Calculations. PROXTIME calculates a single proximity value for each 15 -min interval by the following steps: (1) It scans all entries and sums the number of individuals who report being in each location during this time period. (2) Using the tally calculated for each location in Step 1, it divides the number of individuals who report being in each location by the number of individuals who could have been there (i.e., the total number of individuals in the organization). (3) Using the percentage calculated in Step 2 to weight how proximate individuals are, it multiplies that percentage by the number of individuals who report being in that location. (4) It sums all the weighted values calculated in Step 3. (5) Using the cumulative value arrived at in Step 4, it divides by the total number of people in the organization to yield the average proximity for that period. (6) It repeats Steps 1-5 for each time period.

Hardware and Transportability. The program was developed on an IBM 3081 computer system and is compatible with any computing system that supports a FORTRAN IV compiler.

Program Availability. A copy of the program and additional user-oriented documentation can be obtained at no cost from the second author, c/o Department of Communication, Michigan State University, East Lansing, Michigan 48824.

\section{REFERENCE NOTE}

1. Monge, P. R., White, L., Ejsenberg, E. M., Miller, K. I., 2 Kirste, K. K. The dynamics of organizational proximity. Manuscript submitted for publication, 1982.

\section{REFERENCES}

Conrath, D. W. Communication environment and its relationship to organizational structure. Management Science, 1973, 20, 586-603.

Festivaer, L., Schacter, S., \& Back, K. Social pressures in informal groups: A study of human factors in housing. Stanford, Calif: Stanford University Press, 1950.

KahN, A., \& McG AUaheY, T. A. Distance and liking: When moving close produces increased liking. Sociometry, 1977, 40, 138-144.

Monge, P. R., \& Kinste, K. K. Measuring proximity in human organizations. Social Psychology Quarterly, 1980, 43, 110-115.

Newcoms, T. M. The acquaintance process. New York: Holt, Rinehart, \& Winston, 1961.

Quins, R. Coping with cupid: The formation, impact and management of romantic relationships in organizations. Administrative Science Quarterly, 1977, 22, 30-45.

TEsCH, F. E. Interpersonal proximity and impression formation: A partial examination of Hall's proxemic model. Journal of Social Psychology, 1979, 107, 43-55.

(Accepted for publication November 17, 1982.) 